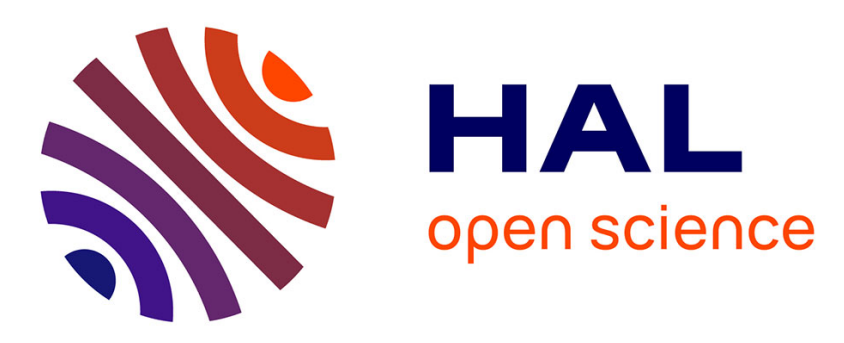

\title{
Heat capacities of mass selected deprotonated water clusters
}

\author{
Sébastien Zamith, Pierre Labastie, Jean-Marc L'Hermite
}

\section{To cite this version:}

Sébastien Zamith, Pierre Labastie, Jean-Marc L'Hermite. Heat capacities of mass selected deprotonated water clusters. Journal of Chemical Physics, 2013, 138 (3), pp.034304. 10.1063/1.4774408 . hal-00776890

\section{HAL Id: hal-00776890 https://hal.science/hal-00776890}

Submitted on 16 Jan 2013

HAL is a multi-disciplinary open access archive for the deposit and dissemination of scientific research documents, whether they are published or not. The documents may come from teaching and research institutions in France or abroad, or from public or private research centers.
L'archive ouverte pluridisciplinaire HAL, est destinée au dépôt et à la diffusion de documents scientifiques de niveau recherche, publiés ou non, émanant des établissements d'enseignement et de recherche français ou étrangers, des laboratoires publics ou privés. 


\title{
Heat capacities of mass selected deprotonated water
}

\section{clusters}

\author{
Sébastien Zamith ${ }^{1,2}$, Pierre Labastie ${ }^{1,2}$, and Jean-Marc L'Hermite ${ }^{1,2}$ \\ ${ }^{1}$ Université de Toulouse ; UPS ; Laboratoire Collisions Agrégats Réactivité, IRSAMC ; F-31062 \\ Toulouse, France \\ ${ }^{2}$ CNRS ; UMR 5589 ; F-31062 Toulouse, France
}

\section{Abstract}

Heat capacities of mass selected deprotonated water clusters $\left(\mathrm{H}_{2} \mathrm{O}\right)_{n-1} \mathrm{OH}^{-}$have been measured in the size range $n=48-118$, as a function of temperature. We have found that they undergo a melting-like transition in the range $110-130 \mathrm{~K}$. The transition temperature is size dependent with a strong correlation with the dissociation energy around the shell closure at $n=55$.

\section{Introduction}

Thermodynamic properties of small particles are not the same as in the bulk. In general, their melting temperatures monotonously decrease with size, essentially owing to the increase of the surface to volume ratio [1]. At the molecular scale, that is for clusters of tens to hundreds atoms or molecules, the size dependence of the thermodynamic properties is more complex. The melting temperature of clusters generally still follows the tendency 
recalled above (with exceptions however, for gallium clusters for instance, whose melting temperature is higher than in the bulk [2]). However, strongly non monotonous size evolution of melting temperatures has been observed for various atomic clusters, together with a similar behavior of the associated latent heats [3].

First order phase transitions can be observed in small clusters by measuring the temperature dependence of their heat capacity (the caloric curve), which exhibits a peak at the transition. This idea has been experimentally used over the last 15 years, since the pioneer work of $\mathrm{H}$. Haberland's group on sodium clusters [3]. Such measurements of melting temperature of mass selected clusters have been extended to a few clusters. All these methods are based on the same principle: cluster's temperature is imposed in a heat bath and their internal energy is measured under the assumption that there is a one-to-one relation, in the canonical ensemble, between cluster's internal energy and their evaporation rate. The evolution of cluster's internal energy $E_{\text {int }}$ as a function of temperature $T$ allows deducing the heat capacity $\frac{d E}{d T}$. The experimental methods used to determine this quantity differ only by the way internal energy is added to the clusters: energy is brought either by a laser [4], or by inelastic [2,5] or attachment [6] collisions.

A first order phase transition is characterized by a peak in the heat capacity. The position of the maximum of the peak indicates the melting temperature, whereas the area of the peak is proportional to the associated latent heat.

The melting temperature of small clusters may vary significantly from one size to another. The main parameter that determine the size dependence of melting temperatures seems to be geometric shell closing [7], but electronic shell closing also plays a role $[8,9]$. The effects 
of the charge state of cluster on the melting temperature and the associated latent heat has been experimentally observed for aluminum clusters [9].

Size dependent heat capacities of protonated and deprotonated water clusters at a temperature of about $155 \mathrm{~K}$ have been reported by Sundén et al [10]. But it is only recently that the temperature dependence of the heat capacity of water clusters, for both negatively [11] and positively [5] charged clusters, has been reported.

The melting transition of water clusters is difficult to investigate: evaporation occurs at a temperature too close to the melting transition and prevents the observation of the whole peak in the caloric curve: from a temperature at which the caloric curve is still rising, evaporation of the clusters occurs before they are probed, which prevents measuring reliably their heat capacity $[5,11]$. Only the observation of a whole peak would be a clear signature of a first order melting transition. However, the caloric curve shows a steep rise, which can reasonably be ascribed to a phase transition $[5,11]$. The position of this sharp change in the caloric curve allows identifying and determining rather accurately a characteristic temperature for this transition. The nature of the corresponding phase transition cannot be deduced from such experiments, it may be either a glass transition or a genuine melting transition [5].

We present in this paper measurements of the caloric curves of deprotonated water clusters. From these curves, transition temperatures are deduced, whose size dependence is studied. It appears that the shell closing at $n=55$ manifests itself as an increase of the transition temperature. The comparison with recent results in the literature $[5,11]$ suggests an effect of the charge state of water clusters on their melting transition. 
This paper is organized as follow: Section II is devoted to the description of the experimental apparatus and an explanation of the method used to deduce the caloric curves is given. In Section III our experimental results are presented and then discussed in section IV. Conclusions will be drawn in section V.

\section{Experimental setup and method}

The experimental setup has already been described in detail in ref [12] and [13]. Water clusters are produced in a gas aggregation source. A discharge in the source allows us the production of ionized species. Under our conditions, we mainly produce protonated $\left(\mathrm{H}_{2} \mathrm{O}\right)_{n} \mathrm{H}^{+}$and deprotonated $\left(\mathrm{H}_{2} \mathrm{O}\right)_{n-1} \mathrm{OH}^{-}$clusters. After their production, clusters are thermalized in a heat bath whose temperature can be controlled down to $25 \mathrm{~K}$.

The clusters are then mass-selected, focused in energy and slowed down [12]. By selecting the polarity of the various voltages, one can study either protonated or deprotonated water clusters. This paper is devoted to the study of the deprotonated ones. After all these preparation steps, the number of molecules $n$ in the cluster is fixed, as well as its initial temperature $T$ and its translational kinetic energy $E_{k}$ in the laboratory frame.

The clusters then enter the collision cell in which a controllable density of water vapor is introduced. When travelling through the cell, the clusters undergo a number of collisions with the monomers of the vapor, leading to attachment and/or evaporation.

After the cell, the products of the collision are mass analyzed with the help of a highresolution time of flight mass spectrometer. The mass spectrum at the output of the cell consists of several peaks corresponding to the intact parent clusters and to clusters that have undergone attachment or evaporation of water molecules. The method used for 
extracting caloric curves from these mass spectra is detailed in ref. 6 . We will only briefly recall it here. For a given temperature of the mass-selected clusters, we record two mass spectra obtained at two different collision energies $E_{c 1}$ and $E_{c 2}$. The barycentres $\bar{n}$ of these mass spectra are then plotted as a function of temperature $T$. The heat capacity is then obtained as [6]:

$$
C(T)=-\bar{\imath}\left(1+\frac{\partial^{2} \bar{n}}{\partial T \partial \bar{\imath}} / \frac{\partial \bar{n}}{\partial T}\right) \frac{\delta E_{t}}{\delta T}
$$

where $\overline{\boldsymbol{l}}$ is the average number of collisions and $\boldsymbol{\delta} \boldsymbol{T}$ is the temperature shift that gives the same barycentre at $\left(T, E_{c 1}\right)$ and $\left(T+\delta T, E_{c 2}\right) . \delta E_{t}$ is proportional to the difference between the two collision energies $\boldsymbol{E}_{\boldsymbol{c} \mathbf{1}}$ and $\boldsymbol{E}_{\boldsymbol{c} \mathbf{2}}$.

In Eq. 1, the term $\frac{\partial^{2} \bar{n}}{\partial T \partial \bar{l}} / \frac{\partial \bar{n}}{\partial T}$ remains small under our experimental conditions. For instance, for the cluster size $\mathrm{n}=54$, the term $\frac{\partial^{2} \bar{n}}{\partial T \partial \bar{l}}$ is about $-5 \cdot 10^{-4}$. The denominator $\frac{\partial \bar{n}}{\partial T}$ varies between $-5.10^{-3}$ at low temperature and $-2.10^{-2}$ at high temperature. Hence this corrective term would at most increase the deduced heat capacities by $10 \%$ at the lowest temperatures and by less than $2.5 \%$ at high temperature. As will be seen later, this correction is clearly within our error bars. This corrective term will only marginally correct our heat capacities but introduces additional noise due to numerical differentiations. Finally we use the following simplified expression:

$$
C(T)=-\bar{\imath} \frac{\delta E_{t}}{\delta T}
$$


The average number of collisions is given by

$$
\bar{\imath}=\rho \sigma v_{\text {rel }} \frac{L_{\text {cell }}}{v_{\text {cluster }}}
$$

where $\rho$ is the density in the cell, $\sigma$ is the collision cross-section, $v_{r e l}$ is the mean relative velocity between the clusters and the single molecules of the vapor, $L_{c e l l}$ is the length of the collision cell and $v_{\text {cluster }}$ the velocity of the clusters in the laboratory frame. We assume the collision cross-section to be given by a hard-sphere model as $\sigma=\pi\left(r_{m} n^{\frac{1}{3}}+r_{m}\right)^{2}$ with $r_{m}=2.25 \AA^{2}$. This is to be taken as an effective value that takes into account electrostatic effects $[13,14]$.

Measurements of attachment cross-sections have shown that only a part $\chi$ of the collisions leads to attachment ( $\chi$ is the ratio of the attachment cross-section to the collision cross-section) $[13,14]$. In the case of attachment, the collision energy is entirely converted into internal energy of the cluster.

For the other inelastic collisions, only a fraction $\alpha$ of the collision energy is converted into cluster's internal energy. Therefore in Eq. $2 \delta E_{t}=\chi \delta E_{c}+\alpha(1-\chi) \delta E_{c}$ where $\delta E_{c}=E_{c 1}-$ $E_{c 2}$. The fraction of collision energy transferred to the cluster can be estimated in the frame of the impulsive collision model $[15,16]$, which gives the value $\alpha=1 / 2$. We take the experimental values of $\chi$ from ref. 14 .

The collision energies are given by: 


$$
E_{c}=\frac{E_{k}}{n+1}+\frac{n}{n+1} k_{B} T_{c e l l} f\left(\frac{E_{k}}{n k_{B} T_{c e l l}}\right)
$$

where $k_{B}$ is the Boltzmann constant, $n$ the size of the cluster, $T_{\text {cell }}$ the temperature of the collision cell and $E_{k}$ the kinetic energy of the clusters in the laboratory frame. The form of the function $f\left(\frac{E_{k}}{n \boldsymbol{k}_{B} T_{\text {cell }}}\right)$ depends on the velocity dependence assumed for the collision crosssection. For a hard sphere cross-section, it goes from 2 for zero velocity clusters up to $5 / 2$ in the limit of clusters having velocities much larger than the molecules. Our experiments are performed in this last limit. If one assumes now a cross-section proportional to $1 / v$ (chargeinduced dipole interaction) or $1 / v^{2}$ (charge-permanent dipole interaction), $f\left(k_{B} T_{\text {cell }}\right)$ is given by $3 / 2$ and $1 / 2$, respectively, in the limit of large clusters velocities. Anyway, the exact form of $f\left(\frac{E_{k}}{n \boldsymbol{k}_{\boldsymbol{B}} \boldsymbol{T}_{\text {cell }}}\right)$ is not crucial: only the difference of collision energies enters in Eq. 1 and the last term in Eq. 4 almost exactly cancels.

As will be shown in the next section, the heat capacities can only be measured up to roughly $140 \mathrm{~K}$. Above this value, evaporation becomes too important to be neglected. We have measured the amount of evaporation by measuring the mass spectra after the collision cell as a function of the initial cluster temperature, with no vapor in the cell. The evaporation is characterized by calculating the proportion of signal appearing in the peaks at $n-1, n-2$, etc.

\section{Experimental Results}


Absolute values of the heat capacity $C(T)$ are measured, in principle, with our experimental method [6]. However, errors may occur in the determination of the absolute value of $\mathrm{C}(\mathrm{T})$, which have several origins. First in the way $\delta T$ is determined. Depending on how the peaks are integrated in the mass spectra and on the way the barycentre curves are smoothed, different values for $\delta T$ may be obtained. Caloric curves have been extracted for different sets of parameters (integration, smoothing) in data processing. Significant sources of uncertainty on the magnitude of the heat capacity come from the multiplying factor $\overline{\boldsymbol{l}}$ and from the energy transferred during the collisions. The estimation of the number of collisions requires the knowledge of the collision cross-section $\sigma$ and a measure of the density in the cell. All in all, we estimate the uncertainty on the determination of the average number of collision to be of the order of $20 \%$.

The simple impulsive collision model we used gives only an estimate of the energy transfer efficiency in inelastic collisions. This model has proven to give reasonable estimates for the energy transfer in nanocalorimetry experiments [17]. Unfortunately, the uncertainty introduced by this model is difficult to quantify.

Finally, the imperfect mass selection of the parent cluster results in small residual peaks in the mass spectra. The influence of these peaks on the determination of the heat capacity is hard to quantify and may vary with temperature. In particular fluctuations of the heat capacity at low temperature must be regarded as experimental noise.

In figure 1 the error bars only include the uncertainties introduced by the data processing used to retrieve $\delta T$ and the uncertainties on the determination of the average number of collisions. The global uncertainty on the absolute value of $C(T)$ is difficult to estimate, but our heat capacities are likely to be at most overestimated or underestimated by a factor of 
2. However, the relative variation with temperature has much smaller uncertainties and the determination of the transition temperature is not significantly affected: indeed, this determination relies on the shape of the heat capacity curve and not on its absolute value.

The heat capacities of deprotonated water clusters $\left(\mathrm{H}_{2} \mathrm{O}\right)_{n-1} \mathrm{OH}^{-}$are presented in Figure 1. for $n=48,53,54,55,56,60,70$ and 118 . The experiments were performed at cluster kinetic energies of $33 \mathrm{eV}$ and $38 \mathrm{eV}$ in the laboratory frame. The water pressure in the cell was 3.8 $10^{-4}$ mbar. On the same figure are shown, when available, the measured evaporation curves. For reference, the heat capacity of bulk ice is also plotted.

The heat capacities present the same features for all sizes: a slow increase with temperature followed by a sudden change in slope. Evaporation sets in relatively rapidly after this change in the heat capacity slope, but clearly at a higher temperature and it is not responsible for the steep rise in the caloric curves. The dashed areas in figure 1 correspond to temperatures for which the amount of evaporation is above $10 \%$. In this region, the initial temperature of the clusters at the entrance of the collision cell is no longer well defined, since some of the mass selected clusters originate in this case from the evaporation of bigger clusters.

From the caloric curves presented in Fig. 1, we extract a transition temperature (the nature of this transition will be discussed in the next section). This temperature is deduced from the point where the slope of the caloric curves changes. This definition of the transition temperature is somewhat arbitrary but allows comparisons from size to size.

The transition temperatures are plotted in the top panel of figure 2 as a function of the cluster size. 


\section{Discussion}

For all the sizes investigated, the heat capacity of deprotonated water clusters is about 1 $\mathrm{k}_{\mathrm{B}} /$ molecule at $100 \mathrm{~K}$, which is roughly a factor of 2 below the heat capacity of bulk ice [18]. No definitive conclusion can be drawn about this factor of 2 given our uncertainties. Above the transition, the heat capacities reaches higher values (up to 6-10 $\mathrm{k}_{\mathrm{B}} /$ molecule) close to the one of bulk liquid water $\left(9 \mathrm{k}_{\mathrm{B}}\right.$ /molecule at ambient temperature). These data are compatible with the experimental values of Hansen and coworkers [10] (see figure 1). For some sizes $(n=55,56)$, they even almost perfectly match. For the other sizes, for which our temperature range doesn't go far enough to establish a direct comparison, the heat capacity that we measure goes significantly higher than the value given by Hansen and coworkers. Although one must be very cautious about this point, it is worth noting that this is compatible with the heat capacity going through a maximum at some point, thus indicating that the heat capacity might present a peak.

The high values attained by the heat capacity indicate that many degrees of freedom have been released at high temperature, indicating an order-to-disorder transition : There is a transition from a rigid to a liquid state, which can thus be called a liquefaction transition. However, the fact that we cannot observe (because of evaporation as explained before) the decrease of the heat capacity after the transition, prevents from definitely ascribing this transition to a real first order melting transition with a well defined corresponding latent heat. An enlightening discussion about glass transition versus melting transition in water clusters can be found in M. Schmidt and B. von Issendorf's paper devoted to the caloric 
curves of protonated water clusters [5]; these authors conclude that it is impossible, using the known techniques for measuring caloric curves, to know whether water clusters undergo a glass transition or a melting transition.

Small water clusters are often supposed to be amorphous in the size range under study here, with a transition between amorphous and crystalline behavior somewhere between $n=200$ and $1000[19,20]$. On the one hand, however, the dissociation energies of deprotonated water clusters obtained by an inversion of mass abundance spectra [21] (see figure 2) show a marked maximum at $n=55$ [22], which may be the signature of a geometrical shell closing. On the other hand, the size evolution of the transition temperatures that we measured clearly exhibits a peak at $n=55$, which is likely to be correlated to the increase of the dissociation energy at this size. The melting temperature of clusters is known to be positively correlated with their dissociation energy $[23,24,25,26]$ : generally, the more stable a cluster, the higher its melting temperature. All these elements put together support the hypothesis of $\left(\mathrm{H}_{2} \mathrm{O}\right)_{54} \mathrm{OH}^{-}$having (in its solid state) an ordered geometry, and thus that it undergoes a melting transition rather than a glass transition. For the other sizes, no conclusion concerning the amorphous or crystalline nature of water clusters can be drawn from the present work.

It is interesting to examine the effect of the charge on the transition temperature. The nature of the charge held by the cluster (protonated, deprotonated, solvated electron) may change its structure, and thus modify the transition temperature, due to different arrangements of the H-bond network. 
The transition temperatures of $\left(\mathrm{H}_{2} \mathrm{O}\right)_{n-1} \mathrm{OH}^{-}$clusters are compared in figure 2 to the ones of $\left(\mathrm{H}_{2} \mathrm{O}\right)_{n}{ }^{-}$[11] and $\left(\mathrm{H}_{2} \mathrm{O}\right)_{n} \mathrm{H}^{+}$species [5], deduced, as in our experiment, from their caloric curve. Schmidt and coworker used a collisionnal excitation technique for measuring the heat capacity of protonated clusters $\left(\mathrm{H}_{2} \mathrm{O}\right)_{n} \mathrm{H}^{+}$in the size range $n=60-79$ [5], whereas the temperature-dependent heat capacity of $\left(\mathrm{H}_{2} \mathrm{O}\right)_{48,118}{ }^{-}$clusters were measured by Hock and coworkers using a laser excitation [11].

The temperature dependence of the heat capacities of protonated clusters $\left(\mathrm{H}_{2} \mathrm{O}\right)_{n} \mathrm{H}^{+}$ shows the same qualitative feature as ours, with a sharp increase at some temperature, which is assigned to a phase transition. Beyond the uncertainty introduced by the two different experimental methods and in spite of possible small discrepancies introduced by the different ways of deducing the transition temperature from the caloric curves, a charge effect seems to exist: The heat capacities of positively charged clusters measured by Schmidt vary monotonically with the size and are systematically higher, by about $10 \mathrm{~K}$, than the few transition temperatures that we measured in the same size range for negatively charged clusters.

It would be of a great interest to measure the transition temperatures of protonated water clusters around the "magic" size $n=55$. By analyzing mass abundance spectra, Hansen and coworkers [21] observed a different behavior for protonated and deprotonated clusters, in the vicinity of this size: they found evidences of shell closing at $n=55$ for deprotonated water cluster whereas for the protonated ones there is no shell closing, but rather a deficit in the stability of $n=56$ : The size evolution of the transition temperature of protonated water clusters is not expected to exhibit the singularity observed around $n=55$ for deprotonated clusters. 
The comparison with experimental transition temperatures of negatively charged clusters $\left(\mathrm{H}_{2} \mathrm{O}\right)_{118^{-}}$and especially $\left(\mathrm{H}_{2} \mathrm{O}\right)_{48}^{-}$is somewhat more surprising. The transition temperatures that we measured for $\left(\mathrm{H}_{2} \mathrm{O}\right)_{47} \mathrm{OH}^{-}(108 \mathrm{~K})$ and $\left(\mathrm{H}_{2} \mathrm{O}\right)_{117} \mathrm{OH}^{-}(124 \mathrm{~K})$ differ from the transition temperatures found by Hock and coworkers for $\left(\mathrm{H}_{2} \mathrm{O}\right)_{48}^{-}(93 \mathrm{~K})$ and $\left(\mathrm{H}_{2} \mathrm{O}\right)_{118^{-}}(118 \mathrm{~K})$. Although the clusters differ only by one hydrogen atom (which might indeed have an effect on the transition temperature) there is in particular a significant difference (by 15K) between the melting temperatures of $\left(\mathrm{H}_{2} \mathrm{O}\right)_{47} \mathrm{OH}^{-}$and $\left(\mathrm{H}_{2} \mathrm{O}\right)_{48}{ }^{-}$. The corresponding caloric curves measured in our experiment and by Hock and coworkers, are shown in figure 3. Our caloric curve exhibits a steep increase which appears at a significantly higher temperature than the same feature in Hock's curve. It is worth noting that Hock's curve shows a second weak inflexion after the first one from which they assign the transition, at a temperature close to our transition temperature. Do the caloric curve of $\left(\mathrm{H}_{2} \mathrm{O}\right)_{48}$ strongly increases again after the highest temperature they investigate? Or do the $\left(\mathrm{H}_{2} \mathrm{O}\right)_{48}^{-}$and $\left(\mathrm{H}_{2} \mathrm{O}\right)_{47} \mathrm{OH}^{-}$cluster have different thermodynamic properties because the nature of the charge is different? $\left(\mathrm{H}_{2} \mathrm{O}\right)_{48}$ is likely to accommodate a solvated electron whereas for $\left(\mathrm{H}_{2} \mathrm{O}\right)_{47} \mathrm{OH}^{-}$the excess electron may be localized on a particular $\mathrm{OH}^{-}$subunit. Could the difference in charge localization induce sufficient structural changes in the clusters that would cause an observable difference in transition temperature? These are open questions.

\section{Conclusion}

The caloric curves of deprotonated water clusters $\left(\mathrm{H}_{2} \mathrm{O}\right)_{n-1} \mathrm{OH}^{-}$have been measured in the size range $n=48-118$. A sharp increase in these curves at a size-dependent temperature is likely to reveal a liquefaction transition. The size evolution of the transition temperatures is strongly correlated with the dissociation energies. In particular, the transition temperature 
at $n=55$ is significantly increased with respect to the neighboring sizes, which is consistent with the hypothesis of geometric shell closing for $\left(\mathrm{H}_{2} \mathrm{O}\right)_{55} \mathrm{H}^{+}$. The transition temperatures of deprotonated clusters are lower than the ones of protonated clusters, and are higher than the transition temperatures of $\left(\mathrm{H}_{2} \mathrm{O}\right)_{n}{ }^{-}$clusters measured by Hock and coworkers.

\section{Acknowledgement}

This work has been partly funded by the Agence Nationale de la Recherche under Grant No. ANR 2011-BS04-028-01.

\section{references}

[1] P. Labastie and F. Calvo, in Thermodynamics and Solid-Liquid Transition, Nanomaterials and Nanochemistry, edited by C. Bréchignac, C. P. Houdy, and M. Lahmani (Springer, New York, 2007)

[2] G. A. Breaux, R. C. Benirschke, T. Sugai, B. S. Kinnear and M. F. Jarrold, Phys. Rev. Lett. 91, 215508 (2003)

[3] M. Schmidt, R. Kusche, B. von Issendorff and H. Haberland, Nature 393, 238 (1998)

[4] M. Schmidt, R. Kusche, W. Kronmüller, B. von Issendorff and H. Haberland, Phys. Rev. Lett. 79, 99 (1997)

[5] M. Schmidt and B. von Issendorff, J. Chem. Phys. 136, 164307 (2012)

[6] F. Chirot, P. Feiden, S. Zamith, P. Labastie, and J.-M. L'Hermite, J. Chem. Phys. 129, 164514 (2008)

[7] H. Haberland, T. Hippler, O. Kostko, M. Schmidt, B. von Issendorf, Phys. Rev. Lett. 94, 035701 (2005)

[8] S. M. Ghazi, M.-S. Lee, and D. G. Kanhere, J. Chem. Phys. 128, 104701 (2008)

[9] A. K. Starace, C. M. Neal, B. Cao, M. F. Jarrold, A. Aguado and J. M. J. Lopez, Chem. Phys. 131, 044307 (2009)

[10] A. E. K. Sundén, K. Støchkel, S. Panja, U. Kadhane, P. Hvelplund, S. Brøndsted Nielsen, H. Zettergren, B. Dynefors and K. Hansen, J. Chem. Phys. 130, 224308 (2009)

[11] C. Hock, M. Schmidt, R. Kuhnen, C. Bartels, L. Ma, H. Haberland, and B. von Issendorff, Phys. Rev. Lett. 103, 073401 (2009)

[12] F. Chirot and S. Zamith and P. Labastie and J.-M. L'Hermite, Rev. Sci. Instrum. 77, 063108 (2006)

[13] S. Zamith, P. Feiden, P. Labastie and J.-M. L'Hermite, J. Chem. Phys. 133, 154305 (2010) 
[14] S. Zamith, P. Feiden, P. Labastie and J.-M. L'Hermite, Phys. Rev. Lett. 104, 103401 (2010)

[15] E. Uggerud and P. J. Derrick, J. Phys. Chem. 95, 1430 (1991)

[16] M. F. Jarrold and E. C. Honea, J. Phys. Chem. 95, 9181 (1991)

[17] C. M. Neal, A. K. Starace and M. F. Jarrold, J. Am. Soc. Mass. Spectrom. 18, 74 (2007)

[18] D. Murphy and T. Koop, Q. J. R. Meteorol. Soc. 131, 1539 (2005).

[19] G. Torchet, J. Farges, M. F. de Feraudy, and B. Raoult, Ann. Phys. Fr. 14, 245 (1989).

[20] Christoph C. Pradzynski et al, Science 337, 1529 (2012)

[21] K. Hansen, P. U. Andersson, and E. Uggerud, J. Chem. Phys. 131, 124303 (2009)

[22] We note that in reference 21 there is a mislabeling of the cluster sizes for the deprotonated species. The number $\mathrm{n}$ should refer to the number of oxygen, not the number of intact water molecules.

[23] H. Haberland, T. Hippler, J. Donges, O. Kostko, M. Schmidt, and B. von Issendorf, Phys. Rev. Lett. 94, 035701 (2005)

[24] E.G. Noya, J. P.K. Doye, and D. J. Wales, Eur. Phys. J. D 43, 57 (2007)

[25] K. Manninen, A. Rytkönen, and M. Manninen, Eur. Phys. J. D 29, 39 (2004)

[26] S. Zamith, F. Chirot and J.-M. L'Hermite, EPL 9213004 (2010) 


\section{Figure captions}

Figure 1: Heat capacities of deprotonated water clusters $\left(\mathrm{H}_{2} \mathrm{O}\right)_{n-1} \mathrm{OH}^{-}$as a function of temperature (thick (red) line) in units of $k_{B}$ per molecule (left scale). The dashed (blue) line is the heat capacity of bulk ice. Also indicated are the measures of Hansen and coworkers [10](stars). The temperature for the heat capacities of Hansen and coworkers are obtained using eq. $A 3$ of ref. 10 together with the dissociation energies of ref. 21 . The percentage of evaporation at the output of the collision cell is plotted as a thin black line (right scale). The dashed area correspond to temperatures for which the evaporation is greater than $10 \%$.

Figure 2: Top panel: Transition temperatures as a function of cluster size. The squares are the results for deprotonated water clusters $\left(\mathrm{H}_{2} \mathrm{O}\right)_{n-1} \mathrm{OH}^{-}$(this work). Results for protonated water clusters $\left(\mathrm{H}_{2} \mathrm{O}\right)_{n} \mathrm{H}^{+}$(circles) and negatively charged clusters $\left(\mathrm{H}_{2} \mathrm{O}\right)_{n}{ }^{-}$are taken respectively from [5] and [11].

Bottom panel: Dissociation energies for deprotonated water clusters taken from [21].

\section{Figure 3:}

(a) Comparison of the caloric curves for deprotonated $\left(\mathrm{H}_{2} \mathrm{O}\right)_{n-1} \mathrm{OH}^{-}$(this work, full lines) and negatively charged $\left(\mathrm{H}_{2} \mathrm{O}\right)_{n}{ }^{-}[11]$ (dashed lines) water clusters for the sizes $\mathrm{n}=48$ (blue) and 118 (red). The full green line is the heat capacity of bulk ice. Also plotted are the points by Hansen and coworkers (stars) [10]. 


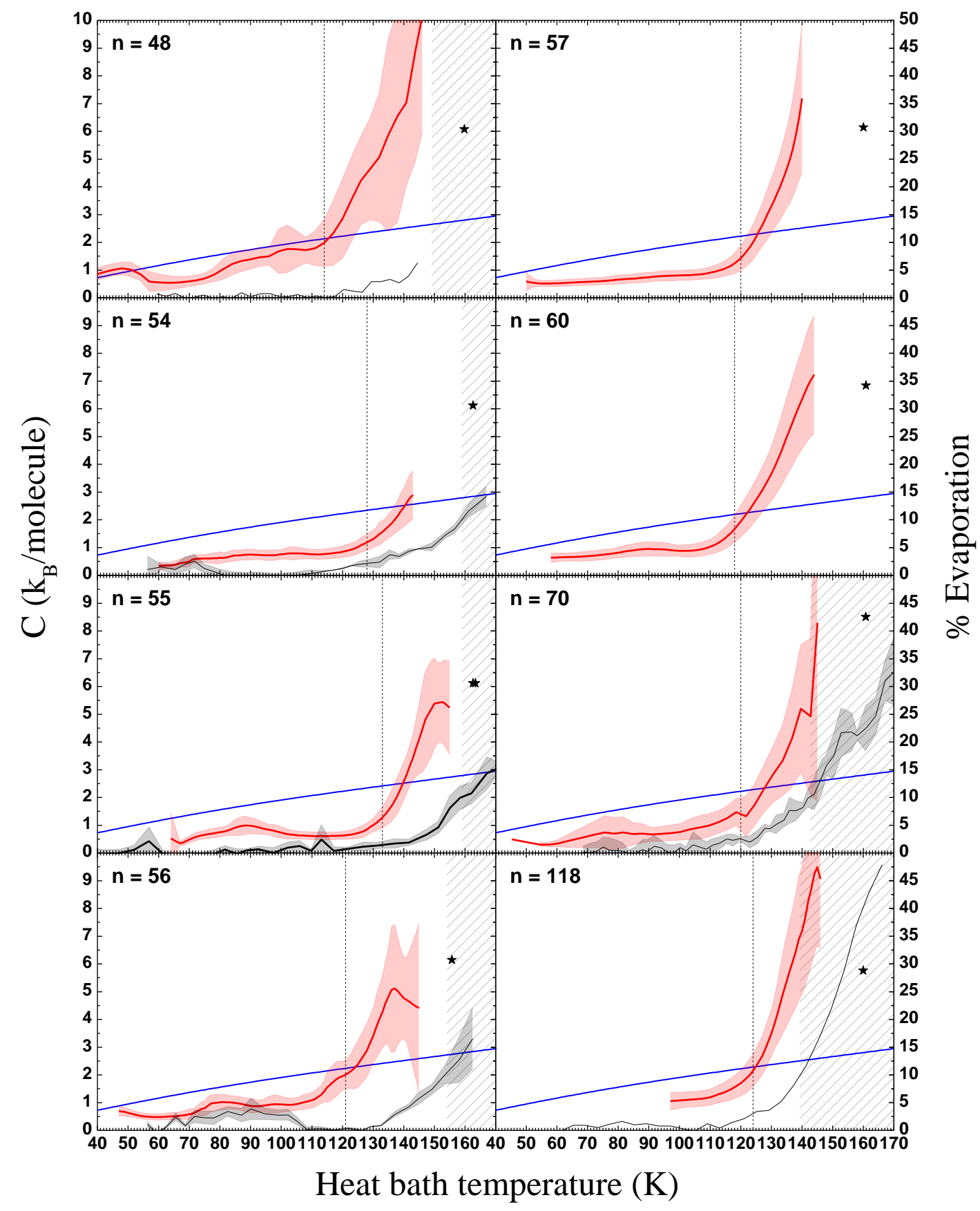

Figure 1 


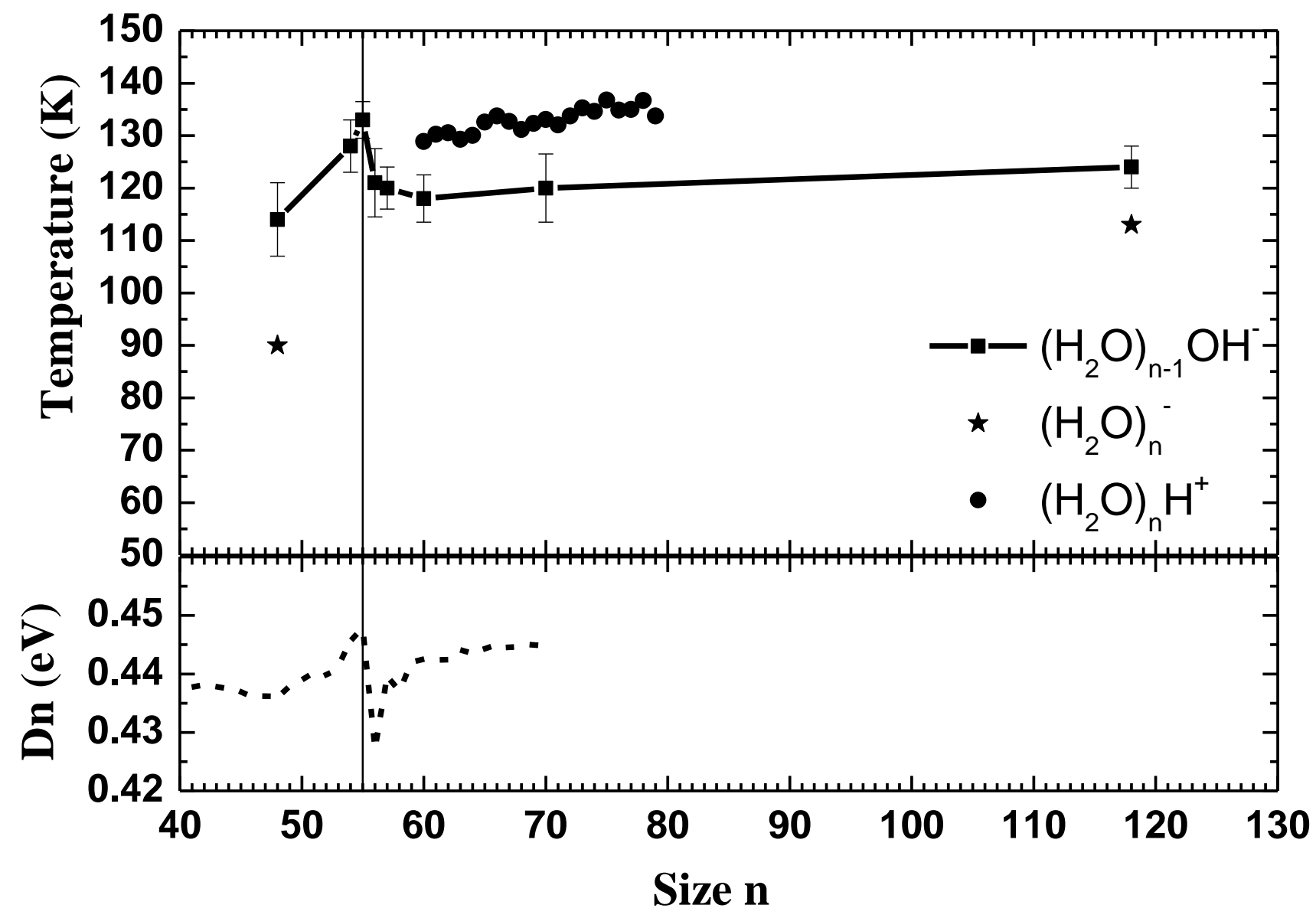

Figure 2 


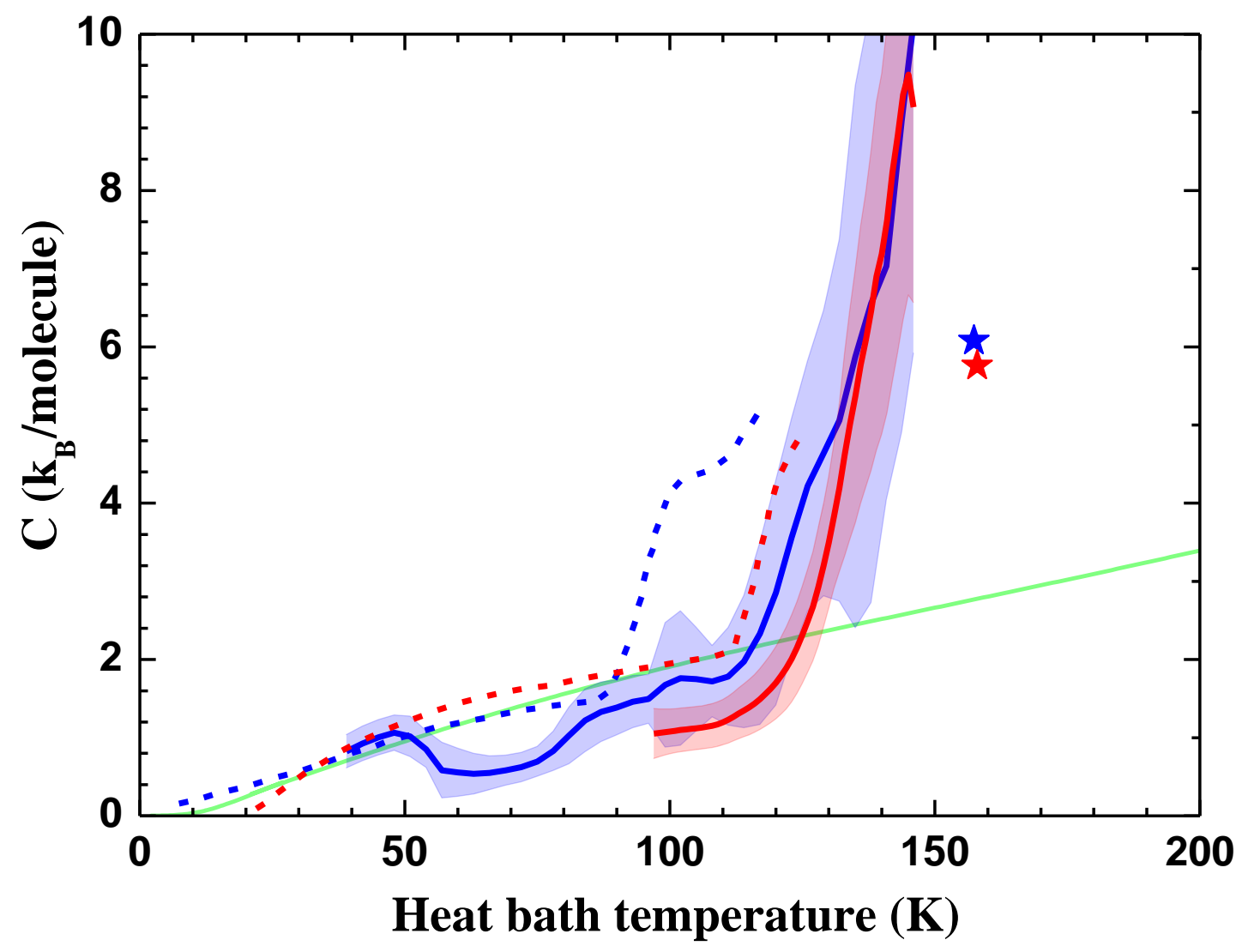

Figure 3 